\title{
Automatic referral to standardize palliative care access : an international Delphi survey
}

\section{Hui, David}

2018-01

Hui , D , Mori , M , Meng , Y-C , Watanabe , S M , Caraceni , A , Strasser , F , Saarto , T, Cherny , N , Glare , P , Kaasa , S \& Bruera , E 2018 , ' Automatic referral to standardize palliative care access : an international Delphi survey ' , Supportive Care in Cancer , vol. 26 , no. 1 , pp. 175-180 . https://doi.org/10.1007/s00520-017-3830-5

http://hdl.handle.net/10138/229880

https://doi.org/10.1007/s00520-017-3830-5

publishedVersion

Downloaded from Helda, University of Helsinki institutional repository.

This is an electronic reprint of the original article.

This reprint may differ from the original in pagination and typographic detail.

Please cite the original version. 


\title{
Automatic referral to standardize palliative care access: an international Delphi survey
}

\author{
David Hui $^{1}$ - Masanori Mori ${ }^{2}$ - Yee-Choon Meng ${ }^{1,3} \cdot$ Sharon M. Watanabe ${ }^{4}$. \\ Augusto Caraceni $^{5}$ • Florian Strasser ${ }^{6} \cdot$ Tiina Saarto $^{7} \cdot$ Nathan Cherny $^{8} \cdot$ Paul Glare $^{9}$. \\ Stein Kaasa ${ }^{10,11} \cdot$ Eduardo Bruera $^{1}$
}

Received: 17 March 2017 / Accepted: 10 July 2017 / Published online: 19 July 2017

(C) Springer-Verlag GmbH Germany 2017

\begin{abstract}
Purpose Palliative care referral is primarily based on clinician judgment, contributing to highly variable access. Standardized criteria to trigger automatic referral have been proposed, but it remains unclear how best to apply them in practice. We conducted a Delphi study of international experts to identify a consensus for the use of standardized criteria to trigger automatic referral.

Methods Sixty international experts stated their level of agreement for 14 statements regarding the use of clinician-based referral and automatic referral over two Delphi rounds. A consensus was defined as an agreement of $\geq 70 \%$ a priori.

Results The response rate was 59/60 (98\%) for the first round and 56/60 (93\%) for the second round. Twenty-six (43\%), 19 (32\%), and $11(18 \%)$ respondents were from North America, Asia/Australia, and Europe, respectively. The panel reached consensus that outpatient palliative care referral should be
\end{abstract}

David Hui

dhui@mdanderson.org

1 Department of Palliative Care, Rehabilitation and Integrative Medicine, Unit 1414, The University of Texas MD Anderson Cancer Center, 1515 Holcombe Boulevard, Houston, TX 77030, USA

2 Palliative Care Team, Seirei Mikatahara General Hospital, Hamamatsu, Shizuoka, Japan

3 Department of Palliative Care, Tan Tock Seng Hospital, Singapore, Singapore

4 Division of Palliative Care Medicine, Department of Oncology, University of Alberta, Edmonton, Alberta, Canada

5 Palliative Care, Pain Therapy and Rehabilitation, Fondazione IRCCS Istituto Nazionale dei Tumori di Milano, Milan, Italy based on both automatic referral and clinician-based referral (agreement $=86 \%)$. Only $18 \%$ felt that referral should be clinician-based alone, and only $7 \%$ agreed that referral should be based on automatic referral only. There was consensus that automatic referral criteria may increase the number of referrals (agreement $=98 \%$ ), facilitate earlier palliative care access, and help administrators to set benchmarks for quality improvement (agreement $=86 \%$ ).

Conclusions Our panelists favored the combination of automatic referral to augment clinician-based referral. This integrated referral framework may inform policy and program development.

Keywords Critical pathways $\cdot$ Delphi technique . Neoplasms $\cdot$ Outpatients $\cdot$ Palliative care $\cdot$ Referral and consultation $\cdot$ Standards
6 Oncological Palliative Medicine, Hematology-Oncology, Cantonal Hospital, St. Gallen, Switzerland

7 Department of Palliative Care, University of Helsinki and Cancer Center, Helsinki University Hospital, Helsinki, Finland Cancer Pain and Palliative Medicine Service, Department of Medical Oncology, Shaare Zedek Medical Center, Jerusalem, Israel

9 Pain and Palliative Care Service, Department of Medicine, Memorial Sloan Kettering Cancer Center, New York, NY, USA

10 European Palliative Care Research Centre, Department of Cancer Research and Molecular Medicine, Norwegian University of Science and Technology, Trondheim, Norway

11 Department of Oncology, Oslo University Hospital and University of Oslo, Oslo, Norway 


\section{Introduction}

Timely involvement of specialty palliative care concurrent with primary palliative care provided by oncology teams has been shown to enhance patient-related outcomes, including symptom control, quality of life, quality of end-of-life care, patient and caregiver satisfaction, and possibly survival $[2,17$, 18]. The growing body of literature has led to endorsement of timely specialty palliative care referral by multiple professional oncology organizations $[3,13,16]$.

While there is consensus that a palliative care referral can benefit cancer patients, many questions remain. When is the optimal time for referral? Who is the ideal subject? And how to standardize the referral process? To address the first two questions, a recent international study by our team identified 11 major consensus criteria and 36 minor criteria for outpatient palliative care referral [10]. However, how these criteria can be put into practice remains to be addressed.

Conceptually, palliative care referral may be initiated by either the oncology team based on clinical judgment or automatic triggers based on pre-defined referral criteria. Currently, referral is predominantly driven by clinicians. Although automatic referral has some theoretical advantages [7], few studies have examined how automatic referral can be applied in practice $[1,5,14]$. A better understanding of this aspect may help to improve patients' access to palliative care. We conducted a Delphi study of international experts to identify a consensus for the use of standardized criteria to trigger automatic referral.

\section{Methods}

\section{Participants}

This study is part of a Delphi survey to examine timebased and need-based referral criteria for outpatient palliative care for patients with advanced cancer, which has been reported recently [10]. The current study focuses on the application of these standardized criteria to trigger an automatic referral. As described previously, we identified international experts based on a literature review on outpatient palliative care, membership involvement in various professional organizations (i.e., the NCCN Palliative Care Guideline Committee, the ESMO Palliative Care Working Group, the Multinational Association of Supportive Care in Cancer (MASCC) Palliative Care study group, and the European Association for Palliative Care), and nominations by the steering committee. These individuals were invited to participate in the Delphi study if they meet the following inclusion criteria: (1) a physician with an active (at least $20 \%$ clinical) specialty clinical practice in either palliative care and/or oncology, with at least
5 years of post-qualification clinical experience; (2) working at a center with outpatient palliative care access; (3) at least one of the following: board certification or equivalent in both oncology and palliative care, have published in the area of integration of palliative care and oncology in the last 10 years, have been involved in national/international palliative care guideline development on the topic of integration; and (4) able to communicate in written English. These criteria were designed to be highly stringent to include only clinicians who have a strong understanding of outpatient palliative cancer care. This study was approved by the institutional review board at MD Anderson Cancer Center with wavier of informed consent.

\section{Survey process}

Web-based surveys were sent to participants using the Qualtrics Survey Software (Qualtrics, Utah, USA) between September 28, 2015 and January 4, 2016. The Delphi study consisted of two rounds separated 6 weeks apart. The duration of each round was 4 weeks. We sent weekly e-mail reminders to non-responders.

We provided the panelists with the following descriptions of clinician-based referral and automatic referral: "Automatic referral involves the use of pre-defined criteria (e.g. diagnosis, prognosis, performance status, symptom intensity, etc) to trigger an outpatient palliative care consultation. This is in contrast to clinician-based referral, which relies on the oncology team to initiate a palliative care referral based on clinician's intuition and/or pre-defined referral criteria. Importantly, automatic referral and clinician-based referral are not mutually exclusive."

We then presented the panelists with 14 statements: nine related to perceived advantages and disadvantages of automatic referral criteria and five on how they may be used in practice. These questions were generated based on literature review and discussions among steering committee members [1, $5,14]$. The full list of questions is shown in Table 1. Their answers were coded on a 5-point Likert scale ("strongly agree," "agree," neither agree nor disagree," "disagree," and "strongly disagree"). They were also given a text box to provide any comments related to automatic referral. A consensus in this study was defined a priori as an agreement (i.e., "Strongly agree" and "agree") by a minimum of $70 \%$ of the experts, consistent with standards in other Delphi studies [6]. We also collected various demographic information from the experts.

The second Delphi round asked the panelists to rate their agreement on the same 14 statements independently and also provide them with the percentage of agreement for each statement from the first round. Because of the stability of findings, we stopped this portion of this study after the second round. 
Table 1 Statements regarding automatic referral

\begin{tabular}{|c|c|c|}
\hline \multirow[t]{2}{*}{ Statements } & \multicolumn{2}{|c|}{$\begin{array}{l}\text { Level of agreement } N \\
(\%)^{\mathrm{a}}\end{array}$} \\
\hline & $\begin{array}{l}\text { First } \\
\text { round }\end{array}$ & $\begin{array}{l}\text { Second } \\
\text { round }\end{array}$ \\
\hline $\begin{array}{l}\text { Automatic referral criteria may increase the number of outpatient palliative care } \\
\text { referrals. }\end{array}$ & $56(95)$ & $55(98)$ \\
\hline $\begin{array}{l}\text { Automatic referral criteria may facilitate earlier palliative care access for cancer } \\
\text { patients. }\end{array}$ & $55(93)$ & $55(98)$ \\
\hline $\begin{array}{l}\text { Automatic referral criteria may help administrators to set benchmarks for quality } \\
\text { improvement. }\end{array}$ & $46(79)$ & $48(86)$ \\
\hline $\begin{array}{l}\text { Automatic referral criteria may result in greater collaboration between oncologists and } \\
\text { palliative care specialists. }\end{array}$ & $35(60)$ & $32(58)$ \\
\hline $\begin{array}{l}\text { Automatic referral criteria may decrease the autonomy of clinicians to deliver primary } \\
\text { supportive/palliative care. }\end{array}$ & $32(56)$ & $29(52)$ \\
\hline $\begin{array}{l}\text { Automatic referral criteria may hinder clinicians' ability to refer patients to palliative } \\
\text { care. }\end{array}$ & $16(28)$ & $5(9)$ \\
\hline $\begin{array}{l}\text { Automatic referral criteria may decrease the number of outpatient palliative care } \\
\text { referrals. }\end{array}$ & $5(9)$ & $1(2)$ \\
\hline $\begin{array}{l}\text { Automatic referral criteria may result in conflicts between oncologists and palliative } \\
\text { care specialists. }\end{array}$ & $30(52)$ & $24(43)$ \\
\hline $\begin{array}{l}\text { Automatic referral mandates routine screening in the oncology clinic which is too } \\
\text { burdensome to conduct regularly. }\end{array}$ & $21(36)$ & $13(23)$ \\
\hline Automatic referral criteria need to be institution specific. & $34(59)$ & $31(55)$ \\
\hline Automatic referral criteria need to be tumor type specific. & $30(52)$ & $25(45)$ \\
\hline $\begin{array}{l}\text { Outpatient palliative care referral should be based on both automatic referral and } \\
\text { clinician-based referral. }\end{array}$ & $50(86)$ & $48(86)$ \\
\hline $\begin{array}{l}\text { Outpatient palliative care referral should be based on automatic referral instead of } \\
\text { clinician-based referral. }\end{array}$ & $16(28)$ & $4(7)$ \\
\hline $\begin{array}{l}\text { Outpatient palliative care referral should be based on clinician-based referral instead of } \\
\text { automatic referral. }\end{array}$ & $10(17)$ & $10(18)$ \\
\hline
\end{tabular}

Items that reached consensus threshold are highlighted in italics

${ }^{a}$ A consensus in this study was defined a priori as an agreement (i.e., "Strongly agree" and "agree") by a minimum of $70 \%$ of the experts. The numbers were italicized if they reached this threshold

\section{Statistical analysis}

We used descriptive statistics to summarize the data. Statistical analysis was conducted with STATA version 12.1 (College Station, TX, USA).

\section{Results}

\section{Response rates and panelist characteristics}

The response rate was 59/60 (98\%) in the first round and 56/ $60(93 \%)$ among the 60 experts participating in this study. Thirty-eight $(63 \%)$ were male, and $32(54 \%)$ were dually trained in both oncology and palliative care. Twenty-six (43\%), $19(32 \%)$, and $11(18 \%)$ respondents were from North America, Asia/Australia, and Europe, respectively. The panelists had a median of 10 years in palliative care (interquartile range [IQR] 7-18.5 years) and 12 years in oncology (IQR 8-20 years).

\section{Perceived advantages and disadvantages associated with automatic referral}

The first and second Delphi rounds showed very similar findings, with the same four of the 14 statements reaching consensus (Table 1). A majority of the panelists believed that automatic referral is associated with both greater number (second round agreement $=98 \%$ ) and earlier referrals (agreement $=98 \%$ ). They can also be useful as benchmarks for quality improvement (agreement $=86 \%$ ).

Several statements did not reach the threshold for consensus. Thirty-two (58\%) believed that automatic referral may result in greater collaboration between oncologists and palliative care specialists; however, 24 (43\%) felt it may result in conflicts between the two teams, 
and $29(52 \%)$ perceived it may hinder clinicians' ability to refer patients to palliative care. Only a small proportion of panelists felt that automatic referral is associated with other negative consequences, such as hindering clinician-based referral $(n=5,9 \%)$, decreasing the number of referrals $(n=1,2 \%)$, or resulting in extra burden for clinicians to conduct $(n=13,23 \%)$.

Several individuals commented on the subtleties of automatic referral:

Difficult balance here, I think there are definitely patients who are missing out on referral due to a range of patient and clinician factors that are well described that may benefit from 'automatic' referral, however a proportion will probably not need referral anyway, and will exacerbate resource constraints which are definitely an issue.

I think triggers may help a clinic get started but, once established, are not necessary. I favor more collaborative and facilitative way of starting the clinic and establishing a practice such as placing the PC provider immediately next to an oncologist in the workroom space and letting the partnership grow naturally. I am, in general, opposed to automatic triggers because they tend to create animosity, open us up to critique based on cost (these consults aren't free), are very atypical in the health system so unfamiliar, often result in patients who are not particularly appropriate, and require a great deal of time explaining why they were "forced" to come and see someone. The set up, even with the patient, can be oppositional - where else in the healthcare system are they required to go and have a consult?

\section{Application of automatic referral in practice}

The panelists reached consensus that outpatient palliative care referral should be based on both automatic referral and clinician-based referral $(n=48$, agreement $=86 \%)$. Only four $(7 \%)$ and $10(18 \%)$ of panelists preferred automatic referral alone or clinician-based referral alone (Table 1).

Our panelists also shared further insights:

Although automatic triggers might be very helpful, they should be adopted through consensus planning among all clinicians involved (especially MDs in both specialties but also other health professionals as well) and their success/value should be reviewed on a regular (quarterly) basis. Thus, even 'automatic triggers' should 'feel' like they are part of the regular clinician-based referral process, otherwise they could be a major source of conflict.
I think that automatic triggers could help physicians highlighting "frail" situations and leading up to a more specific clinician-based evaluation.

\section{Discussion}

Our Delphi panel reached a consensus that automatic referral may improve the volume and timing of palliative care referral. The panelists favored the combination of automatic referral to augment clinician-based referral, instead of either referral model alone. Based on these insights, we proposed a conceptual framework of how automatic referral can be used to augment clinician-based referral.

Currently, clinician-based referral is the predominant path to palliative care access in most centers. The process can be broken down into three steps: (1) recognition of care needs; (2) judging whether this warrants a referral; and (3) initiating a palliative care consultation. Typically, all three steps are conducted by the oncology team, contributing to wide variations in referral because of differences in judgment, time, education, experience, interest, and understanding of palliative care [11, 15]. Although some patients may be referred close to the time of diagnosis, many patients were either not referred or referred within the last days of life, which can significantly diminish the effectiveness of palliative care [9].

To overcome this inequity in palliative care access, automatic referral has been proposed. Under this model, patients care needs are routinely screened, and patients are automatically referred to palliative care if they met the pre-defined criteria. This system-based approach may run parallel and be completely independent to the operations of the oncology team, minimizing human error and judgment. Automatic referral has not been very well tested in clinical practice. A recent survey of $22 \mathrm{NCCN}$ institutions revealed that $3 / 22$ (14\%) inpatient programs and 4/22 (18\%) outpatient programs had automatic referral in place, although the details of the referral process were unavailable [4]. Rocque et al. examined the implementation of triggered universal palliative care consultation for all hospitalized patients with a diagnosis of advanced cancer. The referral rate increased from 12 to $60 \%$ [14]. The vetoing power of the oncology team, inadequate palliative care staffing, and short duration of admission were cited as reasons that not all patients who met the criteria were referred. In a before-after comparison, Adelson et al. examined the application of automatic referral for inpatients and noted a significant increase in the frequency of palliative care referral ( 82 versus $41 \% ; P<0.0001$ ), a reduction in the 30 -day readmission rate (17 versus $36 \% ; P=0.02$ ), and an increase in hospice referral, albeit non-statistically significant (25 versus $14 \% ; P=0.15$ ) [1]. Most recently, our team reported the use of the Edmonton Symptom Assessment Scale for routine 
symptom distress screening in a general oncology outpatient clinic $[8,12]$. Patients who reported three or more symptoms with intensity $\geq 7 / 10$ were assessed by a social worker for triaging. Before and after program implementation, the proportion of highly distressed patients who were referred to palliative care increased from 12 (4 of 34) to $28 \%$ (15 of 58), albeit not statistically significant. More studies are clearly needed to examine the outcomes associated with automatic referral.

Ultimately, few experts felt that clinician-based referral alone nor automatic referral alone would be ideal. Instead, they reached consensus on a combination of both approaches. Exactly how the two approaches should be combined remains to be investigated. Theoretically, referral can be classified as "parallel referral" and "augmented referral". Under the parallel referral model, automatic referral could run independently to clinician-based referral, with the computer generating a palliative care referral for any eligible patients not already referred by the clinical team. Importantly, the standardized criteria used should be agreed upon by all stakeholders (e.g., the oncology team, the palliative care team, and administration). In essence, randomized controlled trials of concurrent palliative care versus routine oncologic care offer insights into how parallel referral compares to clinician-based referral only, demonstrating that parallel referral is associated with greater and earlier palliative care access and improved health outcomes [17, 18]. Alternatively, under the augmented referral model, automatic referral may augment clinician-based referral by alerting the clinician of screening findings, electronically prompting the clinician to refer patients who meet the predefined criteria, and/or asking them for permission to initiate the referral. This method emphasizes a collaborative approach to patient care with system features to enhance practice and may overcome the challenges associated with either referral approaches alone. Because clinicians act as gatekeepers, fewer individuals will likely be referred compared to the parallel referral model [14]. Further research is needed to examine the feasibility and outcomes associated with these two models.

Although randomized trials support that patients with advanced cancer can benefit from a palliative care referral starting around the time of diagnosis, implementation of universal referral is not feasible given the scarce institutional resources. Thus, more selective criteria are needed and would likely need to be customized to each institution based on the existing level of primary and secondary palliative care. An international panel recently identified 11 major criteria on when patients should be referred, which may inform how we can standardize the referral process [10].

This study has several limitations. By design, we included only highly selective experts in this Delphi study who are active clinically and have an in-depth understanding of outpatient palliative cancer care, although their opinions may not reflect that of the greater oncology, primary care, and palliative care communities. Future studies are needed to understand how clinical teams perceive automatic referral in everyday practice. Further research is also required to examine the preferences of patients and family caregivers in regard to how a palliative care referral should be triggered. Developing nations were underrepresented among our panelists, and the process of referral may be different in these countries. This study also focused on outpatient palliative care referral instead of inpatient referral.

In summary, international oncology and palliative care experts identified a consensus for combining automatic referral with clinician-based referral to streamline symptom screening, referral decisions, and referral initiation. We propose parallel referral and augmented referral as two conceptual models operating in between the two extremes of purely clinician-based referral and purely system-based referral. If successfully implemented, these referral models may result in greater number of timely palliative care referral for cancer patients. Further studies are needed to examine the feasibility of their implementation and the impact on referral pattern and patient outcomes.

\section{Compliance with ethical standards}

Funding This study is partly supported by a grant from the Multinational Association of Supportive Care in Cancer. D.H. is supported in part by a National Institutes of Health grant (R21CA186000-01A1), an American Cancer Society Mentored Research Scholar Grant in Applied and Clinical Research (MRSG-14-1418-01-CCE), and an Andrew Sabin Family Foundation Fellowship. We would like to thank all the Delphi panel participants for their expert input.

Conflict of interest The authors declare that they have no conflict of interest.

\section{References}

1. Adelson K, Paris J, Smith CB, Horton J, Morrison SR (2013) Standardized criteria for required palliative care consultation on the solid tumor oncology service. In: Editor (ed)^(eds) Book standardized criteria for required palliative care consultation on the solid tumor oncology service. J Clin Oncol, City, pp. Abstact 37

2. Bakitas M, Lyons KD, Hegel MT, Balan S, Brokaw FC, Seville J, Hull JG, Li Z, Tosteson TD, Byock IR, Ahles TA (2009) Effects of a palliative care intervention on clinical outcomes in patients with advanced cancer: the Project ENABLE II randomized controlled trial. JAMA 302(7):741-749

3. Board IOMNCP (2001) Improving palliative care for cancer. Institute of Medicine, Washington, DC

4. Calton BA, Alvarez-Perez A, Portman DG, Ramchandran KJ, Sugalski J, Rabow MW (2016) The current state of palliative care for patients cared for at leading US cancer centers: the 2015 NCCN Palliative Care Survey J Natl Compr Cancer Netw 14(7): 859-866

5. Doorenbos AZ, Starks H, Bourget E, McMullan DM, LewisNewby M, Rue TC, Lindhorst T, Aisenberg E, Oman N, Curtis JR, Hays R, Clark JD, Baden HP, Brogan TV, Di Gennaro JL, Mazor R, Roberts JS, Turnbull J, Wilfond BS (2013) Examining palliative care team involvement in automatic consultations for children on extracorporeal life support in the pediatric intensive care unit. J Palliat Med 16(5):492-495 
6. Hui D, Bansal S, Strasser F, Morita T, Caraceni A, Davis M, Cherny N, Kaasa S, Currow D, Abernethy A, Nekolaichuk C, Bruera E (2015) Indicators of integration of oncology and palliative care programs: an international consensus. Ann Oncol 26(9):1953-1959

7. Hui $D$, Bruera $E$ (2016) Integrating palliative care into the trajectory of cancer care. Nat Rev Clin Oncol 13(3):159-171

8. Hui D, Bruera E (2017) The Edmonton symptom assessment system 25 years later: past, present and future developments. J Pain Symptom Manag 53:630-643

9. Hui D, Kim SH, Roquemore J, Dev R, Chisholm G, Bruera E (2014) Impact of timing and setting of palliative care referral on quality of end-of-life care in cancer patients. Cancer 120(11):17431749

10. Hui D, Masanori M, Watanabe S, Caraceni A, Strasser F, Saarto T, Cherny N, Glare P, Kaasa S, Bruera E (2016) Referral criteria for outpatient specialty palliative cancer care: an international consensus. Lancet Oncol 17(12):e552-e559

11. Hui D, Park M, Liu D, Reddy A, Dalal S, Bruera E (2015) Attitudes and beliefs toward supportive and palliative care referral among hematologic and solid tumor oncology specialists. Oncologist 20(11):1326-1332

12. Hui D, Titus A, Curtis T, Ho-Nguyen VT, Frederickson D, Wray C, Granville T, Bruera E, McKee DK, Rieber A (2017) Implementation of the Edmonton symptom assessment system for symptom distress screening at a community cancer center: a pilot program. Oncologist. doi:10.1634/theoncologist.2016-0500

13. Levy MH, Smith T, Alvarez-Perez A, Back A, Baker JN, Beck A, Block S, Dalal S, Dans M, Fitch TR, Kapo J, Kutner J, Kvale E, Misra S, Mitchell W, Portman DG, Sauer TM, Spiegel D,
Suzumilowicz E, Taylor RM, Temel J, Tickoo R, Urba SG, Weinstein SM, Zachariah F (2016) NCCN clinical practice guidelines in oncology. Palliative care. In: Editor (ed)^(eds) Book NCCN Clinical Practice Guidelines in Oncology. Palliative Care. National Comprehensive Cancer Network, City

14. Rocque GB, Campbell TC, Johnson SK, King J, Zander MR, Quale RM, Eickhoff JC, Cleary JF (2015) A quantitative study of triggered palliative care consultation for hospitalized patients with advanced cancer. J Pain Symptom Manag 50(4):462-469

15. Schenker Y, Crowley-Matoka M, Dohan D, Rabow MW, Smith CB, White DB, Chu E, Tiver GA, Einhorn S, Arnold RM (2013) Oncologist factors that influence referrals to subspecialty palliative care clinics. J Oncol Pract / Am Soc Clin Oncol 10(2):e37-e44

16. Smith TJ, Temin S, Alesi ER, Abernethy AP, Balboni TA, Basch EM, Ferrell BR, Loscalzo M, Meier DE, Paice JA, Peppercorn JM, Somerfield M, Stovall E, Von Roenn JH (2012) American Society of Clinical Oncology provisional clinical opinion: the integration of palliative care into standard oncology care. J Clin Oncol 30(8):880887

17. Temel JS, Greer JA, Muzikansky A, Gallagher ER, Admane S, Jackson VA, Dahlin CM, Blinderman CD, Jacobsen J, Pirl WF, Billings JA, Lynch TJ (2010) Early palliative care for patients with metastatic non-small-cell lung cancer. N Engl J Med 363(8):733742

18. Zimmermann C, Swami N, Krzyzanowska M, Hannon B, Leighl N, Oza A, Moore M, Rydall A, Rodin G, Tannock I, Donner A, Lo C (2014) Early palliative care for patients with advanced cancer: a cluster-randomised controlled trial. Lancet 383(9930):1721-1730 